\title{
A REVISED ESTIMATE OF THE OCCURRENCE RATE OF TERRESTRIAL PLANETS IN THE HABITABLE ZONES AROUND Kepler M-DWARFS
}

\author{
Ravi kumar Kopparapu ${ }^{1,2,3,4}$
}

\begin{abstract}
Because of their large numbers, low mass stars may be the most abundant planet hosts in our Galaxy. Furthermore, terrestrial planets in the habitable zones (HZs) around M-dwarfs can potentially be characterized in the near future and hence may be the first such planets to be studied. Recently Dressing \& Charbonneau (2013) used Kepler data and calculated the frequency of terrestrial planets in the $\mathrm{HZ}$ of cool stars to be $0.15_{-0.06}^{+0.13}$ per star for Earthsize planets $\left(0.5-1.4 \mathrm{R}_{\oplus}\right)$. However, this estimate was derived using the Kasting et al. (1993) HZ limits, which were not valid for stars with effective temperatures lower than $3700 \mathrm{~K}$. Here we update their result using new HZ limits from Kopparapu et al. (2013) for stars with effective temperatures between $2600 \mathrm{~K}$ and $7200 \mathrm{~K}$, which includes the cool M stars in the Kepler target list. The new habitable zone boundaries increase the number of planet candidates in the habitable zone. Assuming Earth-size planets as $0.5-1.4 \mathrm{R}_{\oplus}$, when we reanalyze their results, we obtain a terrestrial planet frequency of $0.48_{-0.24}^{+0.12}$ and $0.53_{-0.17}^{+0.08}$ planets per M-dwarf star for conservative and optimistic limits of the HZ boundaries, respectively. Assuming Earth-size planets as $0.5-2 \mathrm{R}_{\oplus}$, the frequency increases to $0.51_{-0.20}^{+0.10}$ per star for the conservative estimate and to $0.61_{-0.15}^{+0.07}$ per star for the optimistic estimate. Within uncertainties, our optimistic estimates are in agreement with a similar optimistic estimate from the radial velocity survey of M-dwarfs $\left(0.41_{-0.13}^{+0.54}\right.$, Bonfils et al. $\left.(2011)\right)$. So, the potential for finding Earth-like planets around M stars may be higher than previously reported.
\end{abstract}

Subject headings: planets and satellites: atmospheres

\footnotetext{
${ }^{1}$ Department of Geosciences, Penn State University, 443 Deike Building, University Park, PA 16802, USA

${ }^{2}$ NASA Astrobiology Institute's Virtual Planetary Laboratory

${ }^{3}$ Penn State Astrobiology Research Center, 2217 Earth and Engineering Sciences Building University Park, PA 16802

${ }^{4}$ Center for Exoplanets \& Habitable Worlds, The Pennsylvania State University, University Park, PA 16802
} 


\section{Introduction}

Advances in detection techniques and recent exoplanet surveys have discovered terrestrial planets in the habitable zone (HZ) of their parent star Udrv et al. 2007; Vogt et al. 2010; Pepe et al. 2011a; Borucki et al. 2011; Bonfils et al. 2011; Borucki et al. 2012; Vogt et al. 2012; Tuomi et al. 2012b; Anglada-Escudé et al. 2012) and it is expected that this number will greatly increase as time passes (Batalha et al. 2012). Classically, the HZ is defined as the circumstellar region in which a terrestrial mass planet $\left(0.1 \lesssim M \lesssim 10 \mathrm{M}_{\oplus}\right)$, with favorable atmospheric conditions, can sustain liquid water on its surface (Huang 1959; Hart 1978; Kasting et al. 1993; Underwood et al. 2003; Selsis et al. 2007b; Kaltenegger \& Sasselov 2011; Kopparapu et al. 2013). Currently, more than 800 extra-solar planetary systems have been detected 1 , and $\sim 3000$ additional candidate systems from the Kepler mission are waiting to be confirmed (Batalha et al. 2012).

One of the primary goals of Kepler mission is to determine $\eta_{\oplus}$, the frequency of Earthsize planets in and near the HZ of solar-type stars (Borucki et al. 2011). Such estimates have been made for potentially rocky planets using both radial-velocity (RV, Bonfils et al. (2011)) and Kepler data (Catanzarite \& Shao 2011; Traub 2012; Dressing \& Charbonneau 2013). Furthermore, many other studies have estimated in general the terrestrial planet frequency (Howard et al. 2010; Mayor et al. 2011; Howard et al. 2012; Swift et al. 2012) and the consensus from these studies is that there are more low mass/smaller radii planets than high mass/larger radii ones. Moreover, the frequency increases towards lower radii, larger orbital periods (Howard et al. 2012). The estimates of the occurrence rates of terrestrial planets with periods $<50$ days ranged from $0.23_{-01}^{+0.16}$ (Howard et al. 2010) around GKM stars using RV, to $0.51_{-0.05}^{+0.06}$ (Dressing \& Charbonneau 2013) around M-stars using transit detection.

Specifically, to estimate $\eta_{\oplus}$, one needs to know the boundaries of the HZs. Some studies did estimate this number for M-dwarfs using transit $\left(0.15_{-0.06}^{+0.13}\right.$, Dressing \& Charbonneau (2013)) and RV $\left(0.41_{-0.13}^{+0.54}\right.$, Bonfils et al. (2011)) surveys. Traub (2012) estimated $\eta_{\oplus}$ to be $0.34 \pm 0.14$ for Kepler FGK stars, but this is based on an extrapolation of data for planets with orbital periods shorter than 42 days. These estimates of the occurrence rates were based on 1-D radiative-convective, cloud-free climate model calculations by Kasting et al. (1993). Several other studies (Underwood et al. 2003; Selsis et al. 2007b) parametrized Kasting et al. (1993) results to estimate relationships between HZ boundaries and stellar parameters for stars of different spectral types. Moreover, no uniform criterion of HZ limits were followed in applying them to calculate the occurrence rates, which can lead to com-

1 exoplanets.org 
paring quantities that are not similar. For example, for the outer edge of the HZ, some studies (Dressing \& Charbonneau 2013) used the first $\mathrm{CO}_{2}$ condensation limit and others (Bonfils et al. 2011) used Early Mars criterion.

Recently Kopparapu et al. (2013) obtained new, improved estimates of the boundaries of the HZ by updating Kasting et al. (1993) model with new $\mathrm{H}_{2} \mathrm{O}$ and $\mathrm{CO}_{2}$ absorption coefficients from updated line- by-line (LBL) databases such as HITRAN 2008 (Rothman et al. 2009) and HITEMP 2010 (Rothman et al. 2010). They estimated that, for our Sun, a conservative estimate of the inner $\mathrm{HZ}$ (IHZ) is at $0.99 \mathrm{AU}$ and the outer HZ (OHZ) is at 1.70 AU. These values represent the "water loss" (moist greenhouse) limit at the inner edge and "maximum greenhouse" limit at the outer edge.

Kopparapu et al. (2013) have also estimated HZ boundaries around other stellar spectral types, including $\mathrm{M}$ stars, which are primary targets for ongoing surveys such as Habitable Zone Planet Finder (Mahadevan et al. 2012, HPF) and MEarth (Nutzman \& Charbonneau 2008) to discover potential habitable planets. Furthermore, M-dwarfs are also increasingly becoming important as Kepler targets to find terrestrial size planets: The planetary orbital periods around these stars are shorter, the transit signal is larger, and there is a greater chance of detecting these planets than there is around a Sun-like star. Although M-dwarfs are fainter, the HZs are also closer for M-dwarfs, so it is likely that potential habitable planets may be discovered around nearby M-dwarfs in the near future with space-based characterization missions.

In this study, we apply Kopparapu et al. (2013) HZ limits to estimate the occurrence rate of terrestrial size $\left(0.5-1.4 \mathrm{R}_{\oplus}\right.$ and $\left.0.5-2 \mathrm{R}_{\oplus}\right)$ planets in the HZ of M stars. We base our analysis on Dressing \& Charbonneau (2013) who updated stellar parameters of 3897 low mass Kepler target stars with temperatures below $4000 \mathrm{~K}$ using Darthmouth stellar evolutionary models (Dotter et al. 2008; Feiden et al. 2011). The outline of the paper is as follows: In \$2 we will revise Dressing \& Charbonneau (2013) calculations with new HZ estimates of Kopparapu et al. (2013), discuss the implications in $₫ 3$ and conclude in $₫ 4$.

\section{Estimate of habitable zone planet occurrence rate around M-dwarfs}

From Howard et al. (2012) and Dressing \& Charbonneau (2013), the planet occurrence rate over a given period $(P)$ and radius $\left(R_{p}\right)$ range is given by:

$$
f\left(R_{p}, P\right)=\sum_{i=1}^{N_{p}\left(R_{p}, P\right)} \frac{a_{i}}{R_{\star, i} N_{\star, i}}
$$


where $a_{i}$ is the semi-major axis of planet $i, R_{\star, i}$ is the host star's radius of planet $i, N_{\star, i}$ is the number of stars around which planet $i$ could have been detected and $N_{p}\left(R_{p}, P\right)$ is the number of planets with the radius $R_{p}$ and period $P$. The ratio $a_{i} / R_{\star, i}$ is the inverse of the probability of transit orientation, which is considered to take non-transiting geometries into the estimation of occurrence rate.

In Table 2 of Dressing \& Charbonneau (2013), the authors provide stellar and planetary parameters of candidate KOIs that are considered to be in or near the HZ. To estimate the occurrence rate, Dressing \& Charbonneau (2013) adopt the most conservative estimate of HZ limits from Kasting et al. (1993) results: the 'moist greenhouse' for the inner HZ (0.95 $\mathrm{AU}$ ) and the beginning of the $\mathrm{CO}_{2}$ condensation for the outer $\mathrm{HZ}$ (1.37 AU for the Sun). Furthermore, they consider 'Earth-size' as planets that are in the radius range $0.5-1.4 \mathrm{R}_{\oplus}$. Based on these definitions, Dressing \& Charbonneau (2013) consider two KOIs to be in the HZ from their Table 2: KOI 2626.01 and 1422.02. They then calculate occurrence rate of Earth-size planets in the $\mathrm{HZ}$ as $0.15_{-0.06}^{+0.13}$ planets per star.

This estimate may need to be updated, however, as the estimated width and the position of the HZ has changed recently, following new calculation by Kopparapu et al. (2013)2. According to these authors, HZs are farther out from their star than what has been calculated previously. This will have a significant effect on the occurrence rate derived by Dressing \& Charbonneau (2013). Also, The "1st $\mathrm{CO}_{2}$ condensation" limit of Kasting et al. (1993), should now be disregarded, as it has been shown that $\mathrm{CO}_{2}$ clouds generally warm a planet's climate (Forget \& Pierrehumbert 1997)). Here we use the Kopparapu et al. (2013) HZ limits to derive revised estimates of occurrence rates of potentially habitable planets around low-mass stars. In order to put a lower and upper bound on $\eta_{\oplus}$, we calculate two values for the occurrence rate based on the conservative and optimistic estimate of HZ limits as given in Kopparapu et al. (2013):

(1) In a conservative estimate, the inner edge of the HZ is determined by the "moistgreenhouse" limit which is derived by assuming a fully saturated troposphere and negligible cloud feedback. Neither assumption is likely true in reality, but it is difficult to improve on this with a 1-D climate model because such models cannot accurately simulate clouds or relative humidity. The outer edge of the $\mathrm{HZ}$ is determined by the "maximum greenhouse" limit where a $\mathrm{CO}_{2}$ dominated atmosphere can produce maximum amount of greenhouse warming. Here also, the radiative warming by $\mathrm{CO}_{2}$ clouds is neglected hence the limit is a conservative estimate.

(2) In an optimistic scenario, the inner edge of the HZ boundary can be obtained by the

${ }^{2}$ http://www3.geosc.psu.edu/ ruk15/planets/ 
"recent Venus" limit which is based on the observations of Venus by Magellan spacecraft, suggesting that liquid water has been absent from the surface of Venus for at least 1 Gyr (Solomon \& Head 1991) or earlier. The Sun at that time was $\sim 92 \%$ of the present day luminosity, according to standard stellar evolutionary models (Baraffe et al. 1998; Bahcall et al. 2001, See Table 2). The current solar flux at Venus distance is 1.92 times that of Earth. Therefore, the solar flux received by Venus at that time was $0.92 \times 1.92=1.76$ times that of Earth. This empirical estimate of the inner HZ edge in our Solar System corresponds to an orbital distance of $d=(1 / 1.76)^{0.5}=0.75 \mathrm{AU}$ for the present day. Note that this distance is greater than Venus' orbital distance of 0.72 AU because the constraint of surface water was imposed when the Sun was fainter. The outer edge optimistic estimate is the "Early Mars" limit based on the observation that early Mars was warm enough for liquid water to flow on its surface (Pollack et al. 1987; Bibring et al. 2006). Assuming the dried up riverbeds and valley networks on martian surface are 3.8 Gyr old, the solar luminosity at that time would have been $\sim 75 \%$ of the present value (See Eq.(1) in Gough (1981) and Table 2 in Bahcall et al. (2001)). The present-day solar flux at Mars distance is 0.43 times that of Earth. Therefore, the solar flux received by Mars at $3.8 \mathrm{Gyr}$ was $0.75 \times 0.43=0.32$ times that of Earth. The corresponding empirical OHZ limit for our Solar System today, then, would be $d=(1 / 0.32)^{0.5} \approx 1.77 \mathrm{AU}$.

The optimistic HZ limits can be extrapolated to other stellar types with effective temperatures between $2600 \mathrm{~K}$ to $7200 \mathrm{~K}$, by scaling them with the corresponding values of conservative HZ limits, as shown in Kopparapu et al. (2013).

To derive new rates from Eq.(1), we need two quantities: (1) $a_{i} / R_{\star, i}$ for the planets in the HZs and (2) $N_{\star, i}$, the number of stars around which a planet that has the same size and receives the same insolation as those KOIs in the $\mathrm{HZ}$ could have been detected. The photometrically derived values of $a_{i} / R_{\star, i}$ are given in Table 5 of Dressing \& Charbonneau (2013). But Dressing \& Charbonneau (2013) use the calculated $a_{i} / R_{\star, i}$ determined from the period of the planet and mass of the star (Dressing, private communication). We use the $N_{\star, i}$ values provided within the period-radii cells of Fig. 15 of Dressing \& Charbonneau (2013), which gives the number of stars around which a planet from the center of the grid cell would have been detected with a signal to noise ratio above $7.1 \sigma$. This should still give us nearly the same occurrence rate, or an underestimate of the actual value (see next section). For example, for the two KOIs (2626.01 and 1422.02) that Dressing \& Charbonneau (2013) consider to be in the HZ, when we use the center of the grid cell $N_{\star, i}$ numbers (1822 and 872) from Fig. 15 of Dressing \& Charbonneau (2013) (instead of the $N_{\star, i}$ values provided in section 5.7) and use the period determined $a_{i} / R_{\star, i}$ as they did, we were able to reproduce their value of 0.15 . Thus, our new occurrence rate (which uses center of the grid cell numbers) determined here probably is close to a value from a more rigorous estimate. 
We will first calculate $\eta_{\oplus}$ using Dressing \& Charbonneau (2013) radius range of $0.5-1.4$ $\mathrm{R}_{\oplus}$. Assuming conservative HZ limit 3 from Kopparapu et al. (2013), four KOIs from Table 2 of Dressing \& Charbonneau (2013) should be in the HZ based on the insolation fluxes (Fig.1): KOI 1686.01, 2418.01, 2626.01 and 1422.02. We use the photometric $a_{i} / R_{\star, i}$ from Table 5 of Dressing \& Charbonneau (2013), rather than the calculated $a_{i} / R_{\star, i}$ from the period and stellar mass. For a typical planet candidate, the photometric $a_{i} / R_{\star, i}$ is $85 \%$ of the $a_{i} / R_{\star, i}$ from the period and stellar mass. Therefore, our value tends to be at the lower end of the occurrence rate estimate. The corresponding $a_{i} / R_{\star, i}$ for these candidates from Table 5 of Dressing \& Charbonneau (2013) are: 102.482, 116.837, 36.283 and 51.985, respectively. The corresponding $N_{\star, i}$ values from center of the grid cells of Fig.15 are: 353, 994, 1822 and 872, respectively. Using Eq.(1), we get the conservative estimate of $\eta_{\oplus}$ for low-mass stars to be $0.48_{-0.24}^{+0.12}$ per star.

An optimistic estimate of the occurrence rate can also be derived based on the recent Venus and Early Mars limit from Kopparapu et al. (2013) results. Assuming these limits, six KOIs from Table 2 of Dressing \& Charbonneau (2013) should be in the HZ (Fig.11): KOI 1686.01, 2418.01, 2626.01, 1422.02, 2650.01 and 886.03. The corresponding photometric determined $a_{i} / R_{\star, i}$ for these candidates from Table 5 of Dressing \& Charbonneau (2013) are: $102.482,116.837,36.283,51.985,54.052$ and 39.246 . The $N_{\star, i}$ values from Fig.15 of Dressing \& Charbonneau (2013) are: 353, 994, 1822, 872, 1822 and 2336. Using Eq.(11), the optimistic estimate of the occurrence rate of Earth-size planets in the habitable zones around low-mass stars is $0.53_{-0.17}^{+0.08}$ per star.

Instead of assuming $0.5-1.4 \mathrm{R}_{\oplus}$ as 'Earth-size', we will also calculate occurrence rate extending the radius range from $0.5-2 \mathrm{R}_{\oplus}$. Planets with radius $>1.4 \mathrm{R}_{\oplus}$ are thought to have either homogeneous composition of water ice, silicate or iron or some differential composition of these compounds (Seager et al. 2007; Rogers et al. 2011; Lopez et al. 2012). This will then add KOI 854.01 in the conservative rate estimate (the total number of KOIs in the HZ is then five) and KOI 250.04 in the optimistic rate estimate (the total HZ KOIs is eight). The corresponding $a_{i} / R_{\star, i}$ and $N_{\star, i}$ for these additional candidates from Dressing \& Charbonneau (2013) Table 5 and Fig. 15 are: 90.045 and 2991, respectively, for KOI 854.01; 157.259 and 3287 , respectively, for KOI 250.04 .

Using this expanded definition of Earth-size $\left(0.5-2 \mathrm{R}_{\oplus}\right)$, a conservative estimate of the occurrence rate of Earth-size planets in the HZs around M-dwarfs is $0.51_{-0.20}^{+0.10}$ per star. An optimistic estimate on the occurrence rate is $0.61_{-0.15}^{+0.07}$ per star.

\footnotetext{
${ }^{3}$ ie., Moist-greenhouse limit at the inner edge and maximum greenhouse limit at the outer edge
} 


\section{Discussion}

The occurrence rate estimates derived in the previous section indicate that terrestrial size planets in HZs around low-mass stars are more frequent than previously thought. Couple of caveats are to be noted:

(1) The KOIs are not confirmed planets. The calculated false positive rates for $K e$ pler candidates in our relevant radius bin range from $12.3 \%$ (Fressin et al. 2013) to $\sim 10 \%$ (Morton \& Johnson 2011). Therefore, we may be overestimating $\eta_{\oplus}$. Furthermore, the uncertainties on the fluxes are large (Fig. 1), so some may not be in the HZ (2) On the other hand, We use photometrically derived $a_{i} / R_{\star, i}$ given in Table 5 of Dressing \& Charbonneau (2013). This may underestimate our occurrence rate because Dressing \& Charbonneau (2013) use calculated $a_{i} / R_{\star, i}$ from orbital periods and stellar mass (Dressing 2013, private communication) and the photometric values are $\sim 85 \%$ of the derived values. So the net effect from points 1 and 2 may not change significantly our estimate of $\eta_{\oplus}$.

(3) As mentioned above, we do not calculate number of stars around which a planet that has the same size and receives the same insolation as the ones in the HZ could have been detected; we instead use the cell numbers from Fig.15 of Dressing \& Charbonneau (2013), which gives the number of stars around which a hypothetical planet that has the same radius and period as the center of the grid cell would have been detected with a signal to noise ratio above $7.1 \sigma$. There is no reason to assume that the KOIs considered here to be in the HZ are at the center of the grid cell (in fact, they are not). This offset probably overestimates our value of $N_{\star, i}$ in Eq.(11).

For example, in section 5.7 of Dressing \& Charbonneau (2013), they calculate $N_{\star, i}$ values of 2853, 813 and 2131 for KOIs 854.01, 1422.02 and 2626.01, respectively. These numbers are generally lower in value than the center of the grid cells values we use from Fig.15 (2991, 872, 1822, respectively), except for KOI 2626.01 (an increase of $17 \%$ ). If this is the general trend, i.e, if we are overestimating $N_{\star, i}$ systematically, then our calculated occurrence rates of terrestrial planets in the $\mathrm{HZ}$ around $\mathrm{M}$-dwarfs can be considered as a lower bound to the actual value. Nevertheless, we tested to see how much the rate would change if we change $N_{\star, i}$. It should be noted that $N_{\star, i}$ (at same insolation) is most likely smaller than $N_{\star, i}$ (at same period) for the planet candidates in the HZ because most of those planet candidates orbit stars that are cooler than the typical Kepler M dwarf. Since there is one KOI (2626.01) for which we underestimate $N_{\star, i}$ by $\sim 17 \%$, and could potentially decrease our occurrence rate estimate, we rounded off and added $20 \%$ of the respective $N_{\star, i}$ values to all the KOIs that are in the HZ. After performing this calculation, for the radius range $0.5-1.4 \mathrm{R}_{\oplus}$, the conservative estimate changed from $0.48_{-0.24}^{+0.12}$ to $0.41_{-0.20}^{+0.10}$ per star. The optimistic estimate changed from $0.53_{-0.17}^{+0.08}$ to $0.44_{-0.14}^{+0.07}$ per star. In the extended radius range for Earth-size 
$\left(0.5-2.0 R_{\oplus}\right)$, the conservative estimate changed from $0.51_{-0.20}^{+0.10}$ per star to $0.43_{-0.17}^{+0.08}$, and the optimistic estimate changed from $0.61_{-0.15}^{+0.07}$ per star to $0.52_{-0.13}^{+0.06}$.

Note that even though adding $20 \%$ to $N_{\star, i}$ lowers our occurrence rate, we are underestimating $a_{i} / R_{\star, i}$ on an average of $15 \%$ or more. Furthermore, we showed that we were able to reproduce Dressing \& Charbonneau (2013) estimate even if we use center of the grid cell value for $N_{\star, i}$. Therefore, a more rigorous analysis to determine the occurrence rate from Dressing \& Charbonneau (2013) would likely produce a similar result as our's.

Bonfils et al. (2011) studied 102 southern nearby M dwarfs using ESO/HARPS spectrograph and obtained the frequency of terrestrial mass planets $\left(1-10 \mathrm{M}_{\oplus}\right)$ in the $\mathrm{HZ}$ to be $0.41_{-0.13}^{+0.54}$. Note that they consider two planets to be in the HZ, Gl 581d and Gl 667Cc, using optimistic limits of the HZ (recent Venus and Early Mars) from Selsis et al. (2007b). If we use also use optimistic HZ estimates, we find that both the planets are in the HZ. Our optimistic estimate of $\eta_{\oplus}$ ranges from $0.53_{-0.17}^{+0.08}$ to $0.61_{-0.15}^{+0.07}$ per star, depending on if the mass range in Bonfils et al. (2011) is applicable to terrestrial sizes of $0.5-1.4 \mathrm{R}_{\oplus}$ radius or $0.5-2 \mathrm{R}_{\oplus}$ radius. Nevertheless, our estimates of the occurrence rates of $\mathrm{HZ}$ terrestrial planets around M-dwarfs are in good agreement with independently derived RV estimates, within the error bars.

\section{Conclusions}

The purpose of our analysis is to outline the significant increase in the terrestrial planet occurrence rate in the HZs of M-dwarfs compared to Dressing \& Charbonneau (2013). We have obtained revised estimates based on Dressing \& Charbonneau (2013) estimates of 3987 Kepler M-dwarfs that are cooler than $4000 \mathrm{~K}$ in Q1-Q6 data. Applying new HZ results from Kopparapu et al. (2013) to Earth-size planets $\left(0.5-1.4 \mathrm{R}_{\oplus}\right)$, we calculate that a conservative estimate of the occurrence rate of Earth-size planets in the HZs around M-dwarfs is $0.48_{-0.24}^{+0.12}$ planets per star. The optimistic estimates indicate that the occurrence rate increases to $0.53_{-0.17}^{+0.08}$ planets per star. If we extend the definition of Earth-size to planets in the radius range $0.5-2.0 R_{\oplus}$, the conservative estimate increases to $0.51_{-0.20}^{+0.10}$ per star, and the optimistic estimate increases to $0.61_{-0.15}^{+0.07}$ per star. As discussed in our paper, our results probably are close or underestimate the actual occurrence rate of Earth-size planets in the HZs around M-dwarfs. Furthermore, our optimistic value of $\eta_{\oplus}$ quoted above is in agreement (within uncertainties) with a similar estimate, $0.41_{-0.13}^{+0.54}$, from Bonfils et al. (2011) ESO/HARPS survey of 102 M-dwarf stars indicating that the frequency of terrestrial planets in the HZs of M-dwarfs may be higher than previously reported. 


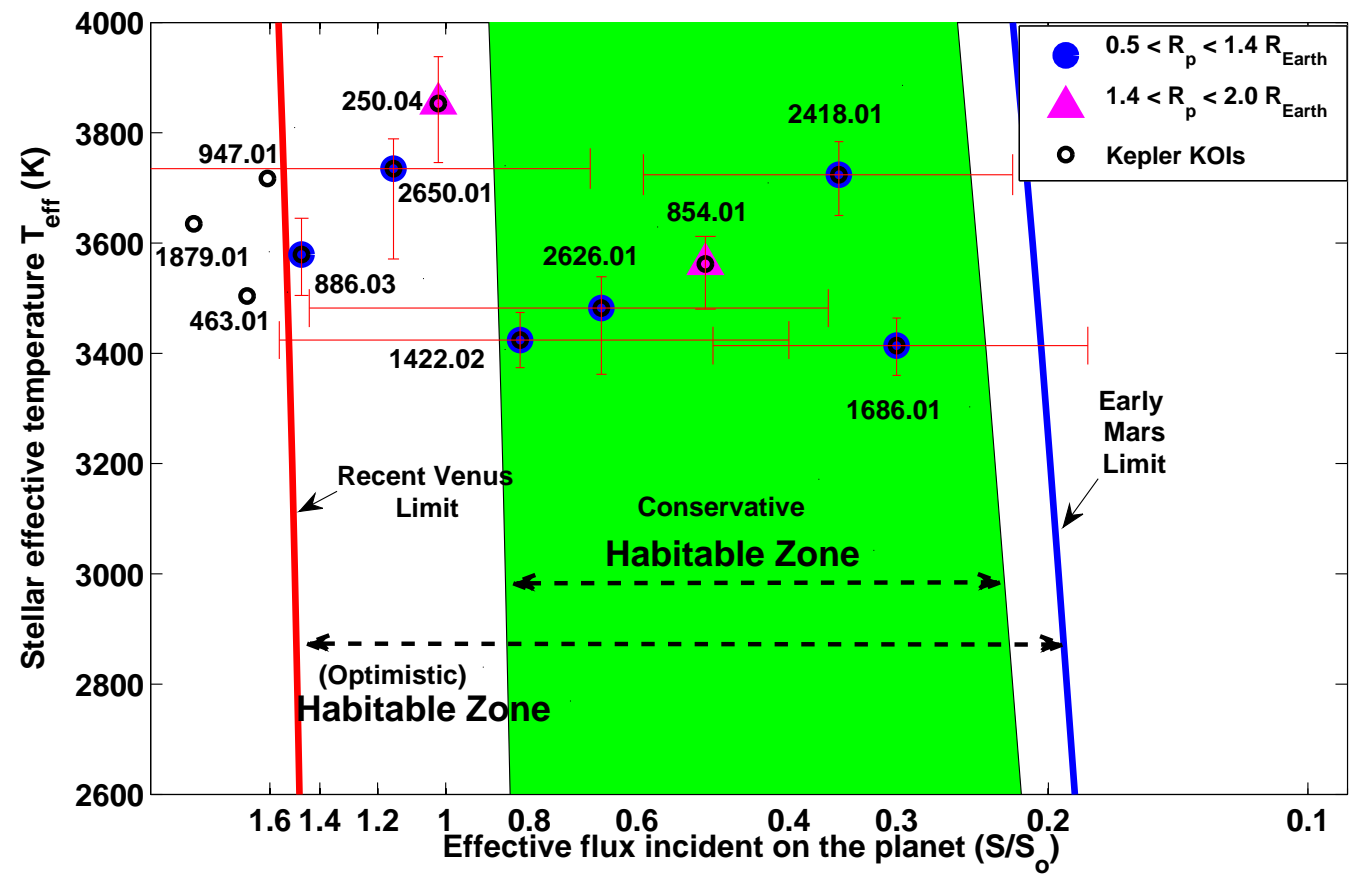

Fig. 1. - Incident stellar flux on a planet as a function of stellar effective temperature, $T_{\text {eff }}$. The green shaded region is the conservative HZ. The optimistic HZ limits are recent Venus (solid red curve) and Early Mars (solid blue curve). Two of the terrestrial-size KOIs (1422.02 and 2626.01) that are in the Dressing \& Charbonneau (2013) HZ are also shown. 
The author is grateful to Courtney Dressing for discussions leading to this paper. The author would also like to thank James Kasting, Steinn Sigurdsson, Eric Feigelson, Suvrath Mahadevan, Jason Wright, Chester Harman, Ramses Ramirez for their valuable input and an anonymous referee whose comments improved the manuscript. R. K, gratefully acknowledge funding from NASA Astrobiology Institute's Virtual Planetary Laboratory lead team, supported by NASA under cooperative agreement NNH05ZDA001C, and the Penn State Astrobiology Research Center.

\section{REFERENCES}

Anglada-Escudé, G., Arriagada, P., Vogt, S. et al. 2012. ApJ, 751, L16

Bahcall, J. N., Pinsonneault, M. H., \& Basu, Sarbani. 2001. ApJ, 555, 990

Baraffe, I., Chabrier, G., Allard, F., \& Hauschildt, P. 1998, A\&A, 337, 403

Batalha, N. M., Rowe, J. F., Bryson, S. T. et al. 2012. submitted to ApJS, arXiv:1202.5852

Bibring, J.-P., Langevin, Y., Mustard, J. F., et al. 2006, Science, 312, 400

Bonfils, X., Delfosse, X., Udry, S. et al. 2011. submitted A\&A, arXiv:1111.5019

Borucki, W. J., Koch, D. G., Basri, G. et al. 2011. ApJ, 736, article id. 19

Borucki, W. J., Koch, D. G., Batalha, N. et al. 2012. ApJ, 745, article id. 120

Catanzarite, J., \& Shao, M. 2011. ApJ, 738, article id. 151

Dotter, A., Chaboyer, B., Jevremovic, D. et al. 2008. ApJS, 178, 89

Dressing, C., \& Charbonneau, D. 2013. ApJaccepted. arXiv:1302.1647

Dumusque, X., Francesco, P., Christophe, L. et al. 2012. Nature, doi:10.1038/nature11572

Feiden, G., A. Chaboyer, B., \& Dotter, A. 2011, ApJ, 740, L25

Forget, F., \& Pierrehumbert, R.T., 1997. Science, 278, 1273

Fressin, F., Torres, G., Charbonneau, D. et al. 2013. arXiv:1301.0842

Gough, D. O. 1981. ESA and European Physical Society, ESLAB Symposium on Physics of Solar Variations, 14th, Scheveningen, Netherlands. Solar Physics, 74, 21

Hart, M. H. 1978, Icarus, 33, 23 
Howard, A. W., Marcy, G. W., \& Johnson, J. A. et al. 2010. Science, 330, 653

Howard, A. W., Marcy, G. W., Bryson, S. T., et al. 2012. ApJ, 201, id. 15

Huang, S. S. 1959, American Scientist, 47, 397

Kasting, J., F., Whitmire, D., P., \& Reynolds. R. T. 1993, Icarus, 101, 108

Kaltenegger, L., \& Sasselov, D. 2011. ApJ, 736, L25

Kopparapu, R. K., Ramirez, R., Kasting, J. F., Eymet, V., Robinson, T. D., Mahadevan, S., Terrien, R. C., Domagal-Goldman, S. D., Meadows, V., \& Deshpande, R. 2013. ApJ, 765, article id. 131

Lopez, E. D., Fortney, J. J., \& Miller, N. 2012. ApJ, 761, article id. 51

Mahadevan, S., Ramsey, L., Bender, C. et al. 2012.To appear in the proceedings of the SPIE2012 Astronomical Instrumentation and Telescopes conference arXiv:1209.1686

Mayor, M., Marmier, M., Lovis, C., et al. 2011. submitted to A\&A, arXiv:1109.2497

Morton, T. D., \& Johnson, J. A. 2011. ApJ, 738, article id 170

Nutzman, P., \& Charbonneau, D. 2008. Publications of the Astronomical Society of the Pacific, 120, 317

Pepe, F., Lovis, C., Segransan, D. et al. 2011a. A\&A, 534, A58

Pollack, J. B., Kasting, J.F., Richardson, S.M., Poliakoff, K., 1987. Icarus, 71, 203

Rogers, L. A., Bodenheimer, P., Lissauer, J. J., \& Seager, S. 2011. ApJ, 738, article id. 59

Rothman, L. S., Gordon, I. E., Barber, A., et al. 2008. JQSRT, 110, 533

Rothman, L. S., Gordon, I. E., Barber, A., et al. 2010. JQSRT, 111, 2139

Selsis, F. et al. 2007b. A\&A, 476, 137

Seager, S., Kuchner, M., Hier-Majumder, C. A., \& Militzer, B. 2007. ApJ, 669, 1279

Solomon, S. C., \& Head, J. W. 1991, Science, 252, 252

Swift, J. J., Johnson, J. A., Morton, T. D. et al. 2012. accepted ApJ, arXiv:1301.0023

Traub, W. A. 2012. ApJ, 745, article id. 20 
Tuomi, M., Jones, H. R. A., Jenkins, J. et al. 2012b. A\&A, accepted arXiv:1212.4277

Udry, S., Bonfils, X., Delfosse, X. et al. 2007. A\&A. 469, L43

Underwood D. R., Jones, B. W., \& Sleep, P. N. 2003. International Journal of Astrobiology, 2,289

Vogt, S. S., Butler, R. P., \& Rivera, E. J. et al. 2010. ApJ, 723, 954

Vogt, S. S., Butler, P., \& Haghighipour, N. 2012. Astronomische Nachrichten, 333, 561

This preprint was prepared with the AAS LATEX macros v5.2. 